\title{
Adrenocortical carcinoma and pulmonary embolism from tumoral extension
}

\author{
Skand Shekhar ${ }^{1}$, Sriram Gubbi², Georgios Z Papadakis ${ }^{3,4}$, Naris Nilubol${ }^{5}$ and Fady Hannah-Shmouni ${ }^{1}$ \\ 1Section on Endocrinology \& Genetics, National Institute of Child Health and Human Development, \\ National Institutes of Health, Bethesda, Maryland, USA, 2Diabetes, Endocrinology, and Obesity Branch, National \\ Institute of Diabetes, Digestive and Kidney Diseases, National Institutes of Health, Bethesda, Maryland, USA, \\ 3Department of Medical Imaging, Heraklion University Hospital, Medical School, University of Crete, Crete, Greece, \\ ${ }^{4}$ Computational Biomedicine Laboratory (CBML), Institute of Computer Science (ICS), Foundation for Research and \\ Technology Hellas (FORTH), Heraklion, Greece, and 5Surgical Oncology Program, National Cancer Institute, National \\ Institutes of Health, Bethesda, Maryland, USA
}

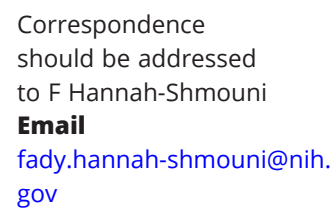

\section{Summary}

Adrenococortical carcinoma (ACC) is a rare cancer, occurring at the rate of one case in two million person years. Cushing syndrome or a mixed picture of excess androgen and glucocorticoid production are the most common presentations of ACC. Other uncommon presentations include abdominal pain and adrenal incidentalomas. In the present report, a 71-year-old male presented with abdominal pain and was eventually diagnosed with ACC. He was found to have pulmonary thromboembolism following an investigation for hypoxemia, with the tumor thrombus extending upto the right atrium. This interesting case represents the unique presentation of a rare tumor, which if detected late or left untreated is associated with poor outcomes, highlighting the need for a low index of suspicion for ACC when similar presentations are encountered in clinical practice.

\section{Learning points:}

- $\mathrm{ACC}$ is a rare but aggressive tumor.

- ACC commonly presents with rapid onset of hypercortisolism, combined hyperandrogenism and hypercortisolism, or uncommonly with compressive symptoms.

- Clinicians should have a low index of suspicion for ACC in patients presenting with rapid onset of symptoms related to hypercortisolism and/or hyperandrogenism.

- Venous thromboembolism and extension of the tumor thrombus to the right side of the heart is a very rare but serious complication of ACC that clinicans should be wary of.

- The increased risk of venous thromboembolism in ACC could be explained by direct tumor invasion, tumor thrombi or hypercoagulability secondary to hypercortisolism.

- Early diagnosis and prompt treatment can improve the long-term survival of patients with ACC.

\section{Background}

The incidence of adrenocortical carcinoma (ACC) is about 1.5-2 cases per million person-years (1). Even though it can present at any age, the age distribution follows a bimodal pattern peaking before the age of 5 and again between the fourth and fifth decades of life, with mean age of diagnosis being 45 years (2). ACC has a female predominance (female:male 1.5-2.5:1) (3).

Approximately $60 \%$ of ACCs are functional, most commonly presenting with isolated Cushing syndrome (45\%) or less commonly as mixed Cushing syndrome and 
virilization (25\%) $(1,4)$. A case of ACC with clinical feature of pheochromocytoma has also been reported (5). Nonfunctioning ACCs (40\%) may present with mass effects and compressive symptoms such as abdominal pain and fullness or, less often, may be discovered incidentally on imaging done for reasons unrelated to adrenal pathology (15\%) (6). The average range in diameter of ACCs is 5-20 $\mathrm{cm}$ and the average weight is $1000 \mathrm{~g}(6,7)$. These tumors are usually highly aggressive, with $50-70 \%$ of them being already in advanced stages upon presentation (8). Most common metastatic sites are lungs (71\%), lymph nodes $(68 \%)$, liver $(42 \%)$ and bone $(26 \%)(6,9)$. Currently, the only curative treatment to ACCs remains aggressive surgical resection. Adjuvant therapies follow the surgery to decrease the risk of recurrence. This approach is the mainstay of treatment mainly for localized ACCs (stages I-III). For the majority of the 'unresectable or metastatic ACCs' all therapy is considered palliative.

\section{Case presentation}

A 71-year-old male was referred for further investigation of an $8 \mathrm{~cm}$ right adrenal mass that was incidentally detected on abdominal computed tomogram (CT) as part of an evaluation for abdominal pain. His comorbities included diabetes mellitus type 2, hypertensive heart disease, anemia and benign prostatic hyperplasia. Physical examination was unremarkable with normal vital signs and a non-cushingoid appearance.

\section{Investigation}

Laboratory data revealed a morning serum cortisol level of 4.9 micrograms/deciliter $(\mu \mathrm{g} / \mathrm{dL})$ following an overnight 1 $\mathrm{mg}$ dose of dexamethasone (using a cut-off of $5 \mu \mathrm{g} / \mathrm{dL}$ ). Serum adrenocorticotropic hormone (ACTH), aldosterone, plasma renin activity, and 24-h urine for metanephrines and normetanephrine were all within normal limits.

The CT imaging revealed a large mass extending from the right adrenal toward superior liver dome with extrinsic pressure on the portal vein, a left upper lobe pulmonary nodule $(1.1 \times 1.1 \mathrm{~cm})$ and a right hilar node $(1.1 \times 0.9 \mathrm{~cm}$; Fig. 1). A CT-guided fine-needle aspiration biopsy of the adrenal mass revealed adrenocortical carcinoma. The whole-body ${ }^{18}$ F-fluorodeoxyglucose positron emission tomograph-computed tomography (PET/CT) scan revealed a large right adrenal mass $(7 \times 6.4 \mathrm{~cm})$ with a maximum standardized uptake value (SUVmax) of 12.4, extending into a large hypermetabolic metastasis in the right hepatic lobe with possible necrotic center (Fig. 1). There were two satellite hepatic lesions adjacent to the mass that had SUVmax of 8.4 and 6.8 and a hypermetabolic $1 \mathrm{~cm}$ left upper lobe lung nodule with an SUVmax of 14.1 (Fig. 1).

On his biweekly follow-up visit to the clinic, the patient complained of recent history of shortness of breath. His vital signs were normal and the oxygen saturation was $99 \%$ on room air. A chest radiograph was unremarkable. Three days later, the patient was hospitalized due to progressive worsening of shortness of breath without complaints of fever, chills, cough or chest pain. Examination revealed normal vital signs (oxygen saturation 98\% on ambient air). Given the suspicion for pulmonary embolism, a CT pulmonary angiography was obtained, which revealed a saddle embolus along with multiple filling defects within the bilateral inferior pulmonary artery branches, and multiple enlarging left upper lobe nodules. A new mass in the right atrium $(5.5 \times 1.7 \mathrm{~cm})$, probably arising from the inferior vena cava, was noted (Fig. 1). A two-dimensional echocardiogram showed left heart failure with an ejection fraction of $22 \%$.

\section{Treatment}

An i.v. heparin drip was initiated. The patient was made 'comfort measures only' after discussion of the poor prognosis with the patient and his family.

\section{Outcome and follow-up}

Follow-up with the pain and palliative care service and social work was arranged.

\section{Discussion}

Adrenal incidentalomas (AIs) are reported in up to $6 \%$ of all abdominal ultrasonographies CT scans performed for various indications, with ACC accounting for $2-5 \%$ of these incidentalomas (10). Most important prognostic factors for both localized and metastatic ACCs are (1) staging; (2) resection status ' $R$ '; and (3) tumor grade (proliferation index, as Ki-67\% and mitotic count) (11).

In case an adrenal mass is incidentally found, evaluation of its malignant potential and hormonal hypersecretion is recommended. Subclinical Cushing's syndrome is reported in $12-16 \%$ of AIs (12). The $1 \mathrm{mg}$ overnight dexamethasone suppression test (DST) is the preferred screening test for subclinical Cushing's syndrome (autonomous glucocorticoid secretion), with an abnormal DST indicating endogenous hypercortisolism. A post-overnight $1 \mathrm{mg}$ DST 8:00 h serum cortisol concentration of $>5 \mu \mathrm{g} / \mathrm{dL}$ establishes the diagnosis of hypercortisolism in the context of AIs. 

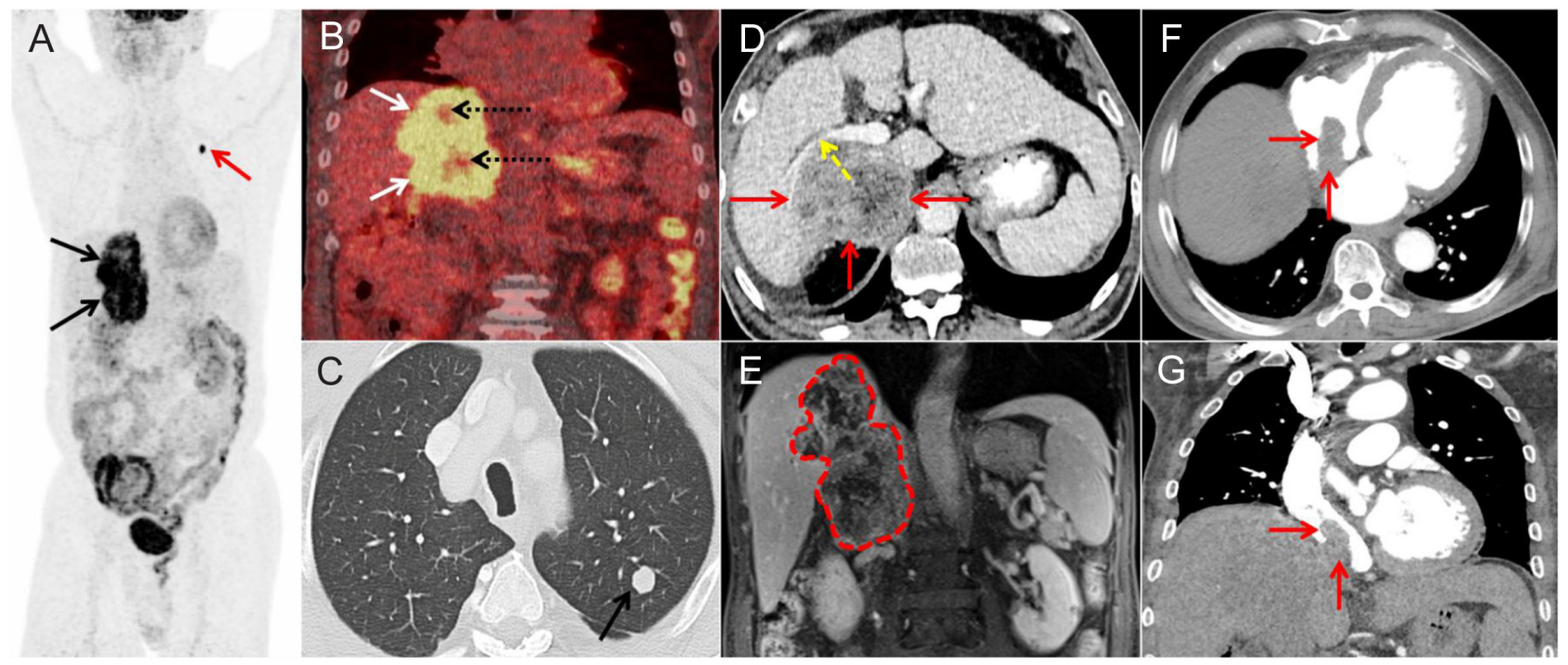

\section{Figure 1}

(A) Whole-body maximum intensity projection (MIP) ${ }^{18} \mathrm{~F}$-fluorodeoxyglucose positron emission tomography - (18F-FDG PET) image showing a large hypermetabolic mass in the right upper quadrant of the abdomen (black arrows) and focal increased uptake in the left upper chest (red arrow). (B) Coronal fused 18F-FDG PET/CT image of the abdomen demonstrating an intensely (SUVmax: 12.4) hypermetabolic right adrenal mass (white arrows) extending superiorly into the liver with photopenic necrotic center (dotted black arrows). (C) Axial CT image of the chest showing a $1 \mathrm{~cm}$ left upper lung lobe metastasis (black arrow), corresponding to the focal lesion seen on the MIP image (A: red arrow). (D) Axial post-contrast CT image of the abdomen demonstrating the extrinsic pressure on the portal vein (dotted yellow arrow) by the mass (red arrows) which arises from the right adrenal gland. (E) Coronal contrast-enhanced T1-weighted fat-suppressed MR image of the abdomen showing (dashed line) the extent of the mass with invasion of the liver and compression effect on the adjacent anatomical structures. ( $F$ and G) Axial and coronal post-contrast $\mathrm{CT}$ images of the chest and upper abdomen showing (red arrows) extension of the right adrenal mass through the inferior vena cava into the right atrium.

Non-functional ACCs are challenging to detect but may present with compressive symptoms such as abdominal fullness and/or pain (in our case), early satiety, nausea or constipation, or an incidental finding on radiographic imaging done for a different reason. The increased risk of venous thromboembolism in ACC could be explained by direct tumor invasion, tumor thrombi or hypercoagulability secondary to hypercortisolism. Rarely, intracaval thrombus extension has been reported and almost all cases were non-functioning ACCs with some reports indicating extension to right atrium or ventricle $(13,14,15)$. Other tumors can also present with IVC thrombus and extension to the right atrum, including metastatic hepatocellular carcinoma (16), colorectal cancer (17), testicular cancer (18), and renal cell cancer (19).

The standard of care for stages I-III ACCs is complete surgical resection, if feasible (11). The status of the resection margins is a major prognostic factor and a Ro resection provides the only means to achieve long-term survival (12). For resectable stage I-III ACCs (European Network for the Study of Adrenal Tumors (ENSAT) criteria), adjuvant mitotane therapy was shown to significantly improve recurrence-free survival $(9,11)$. For reccurent or metastastic ACCs (stage IV, ENSAT criteria), surgical resection can be considered only if two tumoral organs are present, including the adrenal gland (11). Radical surgical resection should be considered in inoperable locally infiltrating or metastatic ACC after reducing tumor burden using neoadjuvant chemotherapy.

Our patient had stage IV disease with metastases to the liver and the lungs. Systemic chemotherapy with etoposide, doxorubicin, and cisplatin, along with mitotane followed by surgery or mitotane monotherapy were initially considered as therapeutic options. However, patient was not interested in receiving the conventional chemotherapy. Unfortunately, further work up for dyspnea revealed multiple enlarging lung nodules and massive pulmonary embolism (8 weeks after initiation of the medication). Tumor thrombus formation and venous migration through the IVC into the right atrium was demonstrated on imaging studies, and this is an exceptionally rare finding, with only few such cases being described in the literature (20).

The approach to management of ACC with atriocaval extension includes adrenalectomy and extraction of the tumor from the inferior vena cava and the right atrium. Early identification of intracaval thrombus using magnetic resnonance imaging (MRI) (preferred imaging modality) is crucial in achieving disease-free survival. The presence of a pulmonary/intracardiac thrombus requires intervention from multiple surgical teams, including endocrine and 
cardiothoracic surgery. For patients who are poor surgical candidates or those who refuse surgery, chemotherapy may be offered. To date, mitotane has been the only drug that has demonstrated efficacy in treating patients with metastatic ACC. The combination of cisplatin, doxorubicin, etoposide and mitotane has produced clinical response rates of about $50 \%$ even in advanced cases (13). In conclusion, ACC can be associated with IVC or intracardiac tumoral extension which can place the patient at risk for pulmonary thromboembolism. Although this is an exceptionally rare finding with ACC, clinicians must be aware of this unique pattern of progression of these tumors and its associated complications.

\section{Declaration of interest}

The authors declare that there is no conflict of interest that could be perceived as prejudicing the impartiality of the research reported.

\section{Funding}

This study was funded by the Intramural Research Program (NICHD, NIDDK and $\mathrm{NCl}$ ) of the National Institutes of Health.

\section{Patient consent}

Consent was obtained from the patient for publication of this case and associated images.

\section{Author contribution statement}

All authors prepared the manuscript. $\mathrm{F} \mathrm{H}$-S provided guidance during the preparation of this manuscript, as well as assistance with the editing process.

\section{References}

$1 \mathrm{Ng}$ L \& Libertino JM. Adrenocortical carcinoma: diagnosis, evaluation and treatment. Journal of Urology 2003169 5-11. (https:// doi.org/10.1097/01.ju.0000030148.59051.35)

2 Stratakis CA \& Chrousos GP. Adrenal cancer. Endocrinology and Metabolism Clinics of North America 200029 15-25, vii-viii. (https:// doi.org/10.1016/S0889-8529(05)70113-4)

3 Xiao XR, Ye LY, Shi LX, Cheng GF, Li YT \& Zhou BM. Diagnosis and treatment of adrenal tumours: a review of 35 years' experience. British Journal of Urology 199882 199-205. (https://doi.org/10.1046/ j.1464-410x.1998.00726.x)

4 Wajchenberg BL, Albergaria Pereira MA, Medonca BB, Latronico AC, Campos Carneiro P, Alves VA, Zerbini MC, Liberman B, Carlos Gomes G \& Kirschner MA. Adrenocortical carcinoma: clinical and laboratory observations. Cancer 200088 711-736. (https:// doi.org/10.1002/(SICI)1097-0142(20000215)88:4<711::AIDCNCR1>3.0.CO;2-W)

$5 \mathrm{Ni} \mathrm{H} \&$ Htet A. Adrenal cortical carcinoma masquerading as pheochromocytoma: a case report. Ecancermedicalscience 20126277. (https://doi.org/10.3332/ecancer.2012.277)
6 Weiss L, Bertagna X, Chrousos G, Kawashima A, Kleihues P, Koch C, Giordano TJ, Medeiros LJ, Merino MJ, Ordonez NG \& Sasano H. Adrenal cortical carcinoma. In: Pathology and Genetics: Tumours of Endocrine Tumors Ed R A DeLelils pp 139-142 2004 Lyon, France: IARC.

7 Icard P, Goudet P, Charpenay C, Andreassian B, Carnaille B, Chapuis Y, Cougard P, Henry JF \& Proye C. Adrenocortical carcinomas: surgical trends and results of a 253-patient series from the French Association of Endocrine Surgeons study group. World Journal of Surgery 200125 891-897. (https://doi.org/10.1007/s00268-001-0047-y)

8 Fassnacht M, Johanssen S, Quinkler M, Bucsky P, Willenberg HS, Beuschlein F, Terzolo M, Mueller HH, Hahner S, Allolio B, et al. Limited prognostic value of the 2004 International Union Against Cancer staging classification for adrenocortical carcinoma: proposal for a revised TNM classification. Cancer 2009115 243-250. (https:// doi.org/10.1002/cncr.24030)

9 Aung SY, Parente P \& McKendrick J. Pulmonary embolism as an initial presentation of adrenocortical carcinoma. World Journal of Oncology 20145 149-152. (https://doi.org/10.14740/wjon814w)

10 Young Jr WF. Clinical practice. The incidentally discovered adrenal mass. New England Journal of Medicine 2007356 601-610. (https:// doi.org/10.1056/NEJMcp065470)

11 Libé R. Adrenocortical carcinoma (ACC): diagnosis, prognosis, and treatment. Frontiers in Cell and Developmental Biology 2015345. (https://doi.org/10.3389/fcell.2015.00045)

12 Berruti A, Baudin E, Gelderblom H, Haak HR, Porpiglia F Fassnacht M, Pentheroudakis G \& ESMO Guidelines Working Group. Adrenal cancer: ESMO Clinical Practice Guidelines for diagnosis, treatment and follow-up. Annals of Oncology 201223 (Supplement 7) vii131-vii138. (https://doi.org/doi:10.1093/annonc/mds231)

13 Hedican SP \& Marshall FF. Adrenocortical carcinoma with intracaval extension. Journal of Urology 1997158 2056-2061. (https://doi. org/10.1016/s0022-5347(01)68152-7)

14 Kumar S, Choudhary GR \& Pushkarna A. Functioning adrenocortical carcinoma with extension upto the right atrium producing Cushing's syndrome. Journal of Clinical Imaging Science 20133 32. (https://doi. org/10.4103/2156-7514.116186)

15 Kim KH, Park JC, Lim SY, Sohn IS, Yun KH, Cho SH, Hong YJ, Park HW, Kim JH, Kim W, et al. A case of non-functioning huge adrenocortical carcinoma extending into inferior vena cava and right atrium. Journal of Korean Medical Science 200621 572-576. (https:// doi.org/10.3346/jkms.2006.21.3.572)

16 Quencer KB, Friedman T, Sheth R \& Oklu R. Tumor thrombus: incidence, imaging, prognosis and treatment. Cardiovascular Diagnosis and Therapy 20177 (Supplement 3) S165-S177. (https:// doi.org/10.21037/cdt.2017.09.16)

17 Meyers D, Nixon NA, Franko A, Ng D \& Tam VC. Tumour thrombus of the inferior vena cava extending into the right atrium in the setting of colon cancer. BMJ Case Reports 2017 2017. (https://doi. org/10.1136/bcr-2016-218107)

18 Geffen DB, Kaneti J, Hendler N \& Hertzanu Y. Testicular carcinoma with inferior vena cava thrombosis extending into the right atrium treated with chemotherapy and anticoagulation. European Urology 199221 82-84. (https://doi.org/10.1159/000474807)

19 Oltean MA, Matuz R, Sitar-Taut A, Mihailov A, Rednic N, Tantau A, Toganel R, Minciuna IA, Orasan O, Muresan F, et al. Renal cell carcinoma with extensive tumor thrombus into the inferior vena cava and right atrium in a 70-year-old man. American Journal of Men's Health 2019 13. (https://doi.org/10.1177/1557988319846404)

20 Rosen B, Rozenman Y \& Harpaz D. Extension of adrenocortical carcinoma into the right atrium - echocardiographic diagnosis: a case report. Cardiovascular Ultrasound 20031 5. (https://doi. org/10.1186/1476-7120-1-5)

Received in final form 29 October 2019

Accepted 5 November 2019 